Association for Information Systems

AIS Electronic Library (AISeL)

Wirtschaftsinformatik 2021 Proceedings

Track 11: Human Computer Interaction

\title{
Curing Toxicity - A Multi-Method Approach
}

\author{
Bastian Kordyaka \\ University of Siegen \\ Björn Kruse \\ South Westphalia University of Applied Sciences
}

Follow this and additional works at: https://aisel.aisnet.org/wi2021

Kordyaka, Bastian and Kruse, Björn, "Curing Toxicity - A Multi-Method Approach" (2021).

Wirtschaftsinformatik 2021 Proceedings. 6.

https://aisel.aisnet.org/wi2021/PHuman/Track11/6

This material is brought to you by the Wirtschaftsinformatik at AIS Electronic Library (AISeL). It has been accepted for inclusion in Wirtschaftsinformatik 2021 Proceedings by an authorized administrator of AIS Electronic Library (AISeL). For more information, please contact elibrary@aisnet.org. 


\title{
Curing Toxicity - A Multi-Method Approach
}

\author{
Bastian Kordyaka $^{1}$ and Björn Kruse ${ }^{2}$ \\ ${ }^{1}$ University of Siegen, Chair of Information Systems, Siegen, Germany \\ \{bastian.kordyaka\}@uni-siegen.de \\ ${ }^{2}$ South Westfalia University of Applied Science, Meschede, Germany \\ \{kruse.bjoern\}@fh-swf.de
}

\begin{abstract}
Enabled by technological advancements, a contemporary form of technology use that particularly became popular are online multiplayer video games, which are played with others in real time. Besides various positive impacts on the user experience (e.g., fun, additional social exchange) adverse consequences have occurred as well (e.g., stress, anger). Most recently, a sincere problem gaining increased attention is toxic behavior (i.e., a behavior spreading negative effects and bad mood during play). With our study, we propose a way to handle toxic behavior on a level of video game design by using a multi-method approach. First, we will consult the online disinhibition effect and its antecedents to identify design related relationships. Afterwards, we will conduct a qualitative workshop engaging video game designers and players to reshape in-game experiences by incentivizing players to buffer toxicity.
\end{abstract}

Keywords: User experience engineering, toxic behavior, online disinhibition effect, multi-method approach.

\section{Introduction}

Accompanied by the widespread digitalization during the last decades, new forms of technology use emerged. One such instance is computer-mediated communication (CMC), which occurs through the use of technological devices supporting the communication of individuals [1]. Besides various beneficial consequences (e.g., effective communication, location independent work) the dissemination of CMC had some adverse impacts on health and well-being of individuals (e.g., stress and strain mediated through technology) [2,3].

One form of technology use that became particularly popular and is driven by CMC are online multiplayer video games (OMGs). They are collaboratively played in real time over the internet with other players around the world. Collaboratively playing games online is an important leisure activity in times of the world-wide corona pandemic, as it creates opportunities to establish additional forms of social exchange largely missing in real life. In this context, a phenomenon that already has attracted the attention of academia [4-6] and practice [7] is toxic behavior (TB). TB can be understood as a form of aggressive behavior towards members of the own team, which contributes to an increasingly negative affect and bad mood during gameplay [6]. 
Currently, TB is considered one of the sincerest problems and a main driver of the churn of players in OMGs that largely remains unsolved [8].

With our study, we seek to utilize the (unexploited) potential of video game design to buffer TB. For this, we refer to present findings that showed substantial influences of the online disinhibition effect (ODE) as a predictor of TB [8] and identified antecedent variables of the ODE indicating its potential to be addressed on the level of game design (i.e., dissociative anonymity, invisibility, asynchronicity, solipsistic introjection, dissociative imagination, and minimization of authority) [9]. Specifically, we propose the application of a multi-method approach. First, we want to identify meaningful ODE antecedent variables explaining TB using a quantitative survey. Second, we will use the derived information to qualitatively explore manifestations on the level of video game design elements. By carrying out a workshop with various stakeholders (e.g., game designers, social and computer scientists) to identify mechanisms to buffer TB. Our paper is guided by the subsequent research questions:

RQ1: What antecedent variables related to the ODE explain TB?

RQ2: What design elements hold the potential to reduce the occurrence of TB?

With our short paper, we seek to make the following contributions. First, it will allow academia to better understand the occurrence of TB and its influences of a new class of potential predictor variables (ODE antecedents), opening up a variety of subsequent research questions regarding new forms of CMC and aggressive behavior online. Second, it provides practical implications for the video game industry to better handle TB. This substantially improves the user experience for their communities and prevent the churn of players significantly lowering profit margins.

\section{$2 \quad$ Related Work}

\subsection{Video Games as Popular Form of Technology Usage}

Enabled through the technological advancements during the last decade, OMGs disrupted the market of video games regarding popularity and revenue, whereby this development is closely linked to the meaningfulness of competitive multiplayer games $[10,11]$. OMGs such as League of Legends (LoL) or Fortnite allow thousands of people around the world to interact with each other in real-time and can be considered elementary pillars of the contemporary digital culture. As an example, LoL has more than 115 million active players and made around $\$ 1.4$ billion revenue in $2018[15,16]$. For the purpose of our paper, we refer to LoL because of its team-based design and high levels of interactivity between players. As a consequence, toxic behavior can be considered an ordinary form of behavior that occurs in almost every game. This allows us to holistically explore toxicity in one of the most popular multiplayer contexts [17]. 


\subsection{The Adverse Impact of Toxic Behavior}

For the purpose of our paper, we understand TB as an aggressive behavior that occurs when a video game player comes across a negative event during a game, that generates anger and frustration, leading to a harmful, contaminated, and disseminated toxic type of communication using pings and text chat primary towards members of the own team [6]. Manifestations of TB are behaviors such as flaming, trolling, or insulting other players. Moreover, TB is a genuine problem in various OMGs because the necessity for players to cooperate and interact with each other in real time to win becomes undermined. As a consequence, TB scares away new players and leads to the churn of existing players. Opposed to related constructs of online aggressive behavior on the internet such as cyberbullying, TB occurs in a rather short period of time (the ordinary game length is around 30 minutes) and can be understood as state variable that can be executed by a single individual (in the case of cyberbullying more than one perpetrator is necessary). Additionally, TB is an ordinary part of the relevant gaming cultures, which underlines its wide dissemination. Taken together, TB remains a sincere problem for different video game communities. While previous research occasionally dealt with TB and it is still unclear how to holistically buffer toxicity from a theoretical standpoint on a level of game design $[8,18,19]$.

\subsection{Online Disinhibition Antecedents}

Disinhibition can be understood as the perceived lack of restraint an individual feels when communicating online compared to communicating in-person, due to decreased behavioral inhibitions [9]. Usually, the ODE is operationalized with two different dimensions: benign disinhibition (i.e., positive behavior such as helping others, and showing kindness) and toxic disinhibition (i.e., negative behavior such as hostile expressions, and inappropriate behaviors). In previous research, the ODE already illustrated its potential to explain aggressive behavior on the internet [20-23]. Referring to the context of $\mathrm{TB}$, a recent study showed the meaningfulness of toxic disinhibition as a predictor for TB [8]. Additionally, various antecedent variables of the ODE were identified: dissociative anonymity (e.g., perceived anonymity), invisibility (e.g., not being physically visible), asynchronicity (e.g., communication does not happen in realtime), solipsistic introjection (e.g., self-constructed characteristics of others), dissociative imagination (e.g., being in an alternative reality), and minimization of authority (e.g., reduced responsibility). We believe that the antecedent variables are particularly suitable to be addressed on a level of game design influencing both dimensions of disinhibition. To the best of our knowledge no study tried to explore the influences of the ODE variables as predictors of TB, which is a knowledge gap we seek to close with our study. 


\section{$3 \quad$ Methodology}

\subsection{Research Design and Data Analysis}

To answer our two research questions, we plan to carry out a multi-method approach using quantitative and qualitative methods respectively, which will give us the opportunity to derive unique and connecting aspects of knowledge. First, we want to better understand influences of the ODE antecedent variables during the occurrence of TB using co-variance-based statistics. Since the main goal of our study is the identification of opportunities to buffer TB on a level of game design and not the development or continuation of existing theories, we decided out of reasons of parsimony to not specify concrete hypotheses for our quantitative analysis because we understand it as an initial and explorative step of our multi-method approach. Second, we want to use the derived information from the quantitative part and conduct a moderated focus group workshop engaging game designers and players to designate design elements to buffer TB. For this, we will define and explain the most important antecedent variables as a framework condition. The workshop will consist of a semistructured narrative phase with pre-formulated questions and a discussion phase to identify additional design elements following a structured procedure from previous research [24, 25]. Its main goal is to derive design principles based on the relevant predictors of our quantitative study, to buffer TB on a level of game design. Additionally, we plan to develop a heuristic prototype that addresses different usage scenarios (e. g. pre-game/post-game, in-game, and game client).

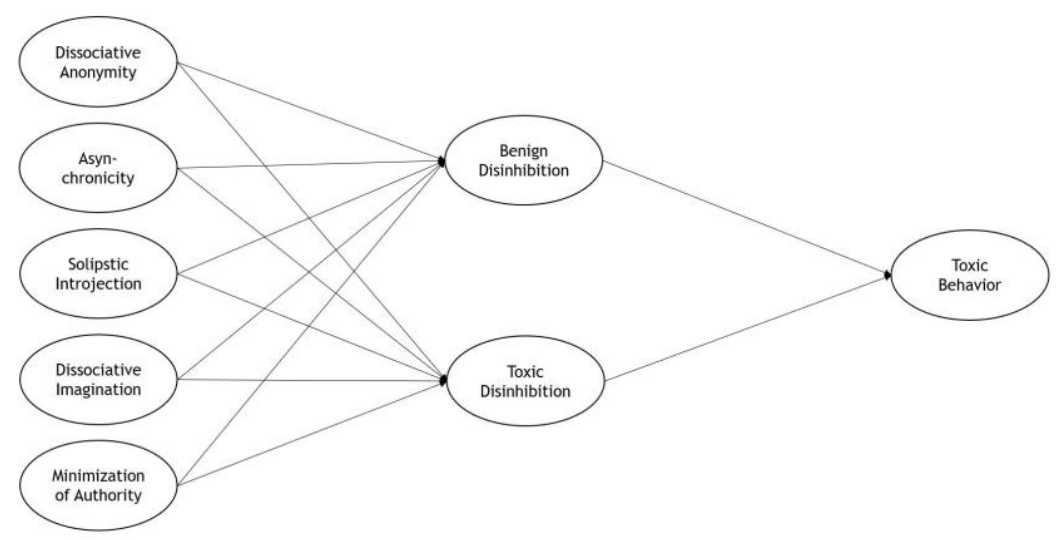

Figure 1. Research Model of the Quantitative Approach

\subsection{Participants and Data Collection}

We will collect two different data samples. First, we use an online survey to ask individuals who frequently play LoL about variables related to the ODE as explanations for TB. In order to acquire a significant number of respondents $(\mathrm{N}>300)$, we will use the crowdsourcing marketplace Mechanical Turk. Second, we will recruit a sample (N 
> 10) consisting of different groups of stakeholders who are familiar with the design of video games (i.e., technology development, game designers, and social and computer sciences) to participate in our workshop. Accordingly, we will derive a convenient sample of respondents from the internal and external networks of our institution, while ensuring a maximum of diversity regarding academic background and demographics.

\subsection{Quantitative Measurements}

To measure the quantitative constructs in our study, we will use empirically validated scales adjusted to the context of our study. The majority of scales will use a seven-point Likert scale ranging from 1 ("strongly disagree") to 7 ("strongly agree"). Additionally, we will measure various demographic (e.g., age, sex, education, origin) and control variables (e.g., enjoyment, hours of play, experience of play, game type) to prevent unwanted confounding effects.

Dependent variable: Toxic behavior will be measured by using five items from (e.g., "If I get mad during a game, I insult others") [4].

Mediating variables: We plan to use an existing scale to measure ODE: benign disinhibition will be measured with six items (e.g., "I have an image of the other players in my head when I read their messages") and toxic disinhibition with four items (e.g., "There are no rules online therefore you can do whatever you want") [21].

Independent variables: We will use a scale comprising of thirty-eight items to measure the ODE antecedent variables, which consists of the dimensions dissociative anonymity (e.g., "I can hide my identity"), invisibility (e.g., "My actions are invisible"), asynchronicity (e.g., "I can control the pace of communication"), sollipstic introjection (e.g., "I assign a character to that player I am communicating with."), dissociative imagination (e.g., "My game related life is separated from the offline world") and minimization of authority (e.g., "I can get rid of authority") [26].

\section{Conclusion and Outlook}

Embedded in the assumptions of the ODE, in this short paper we propose a multimethod approach to identify design elements to buffer TB in OMGs. For this, we outlined our two-step approach and how we plan to identify and use the potential of the ODE variables to buffer toxicity in OMGs. Specifically, we will derive design principles based on the relevant predictors of our quantitative study to buffer TB on a level of game design and develop a heuristic prototype that can be used in subsequent experimental research. Academia can build on the findings, integrate them within existing frameworks, and pursue the derived body of knowledge. Developers and video game designers can utilize the findings to better handle TB and substantially improve the user experience of OMG players resulting in larger player bases and increased revenues eventually. 


\section{References}

1. McQuail, D.: McQuail's Mass Communication Theory. Sage publications (2010).

2. Tarafdar, M., Tu, Q., Ragu-Nathan, B.S., Ragu-Nathan, T.S.: The Impact of Technostress on Role Stress and Productivity. Journal of Management Information Systems. 24, 301-328 (2007).

3. Fuglseth, A.M., Sørebø, Ø.: The Effects of Technostress Within the Context of Employee Use of ICT. Computers in Human Behavior. 40, 161-170 (2014).

4. Kordyaka, B., Klesel, M., Jahn, K.: Perpetrators in League of Legends: Scale Development and Validation of Toxic Behavior. In: Proceedings of the 52nd Hawaii International Conference on System Sciences, pp. 1-10 (2019).

5. Blackburn, J., Kwak, H.: STFU NOOB!: Predicting Crowdsourced Decisions on Toxic Behavior in Online Games, ACM Press, pp. 877-888 (2014).

6. Neto, J.A.M., Yokoyama, K.M., Becker, K.: Studying Toxic Behavior Influence and Player Chat in an Online Video Game. In: Proceedings of the International Conference on Web Intelligence. pp. 26-33 (2017).

7. Fair Play Alliance, http://www.fairplayalliance.org/, last accessed 2020/04/26.

8. Kordyaka, B., Jahn, K., Niehaves, B.: Towards a Unified Theory of Toxic Behavior. Internet Research (2020).

9. Suler, J.: The Online Disinhibition Effect. Cyberpsychology \& Behavior. 7, 321-326 (2004).

10. Martinello, League of Legends generated $\$ 1.5$ billion revenue in 2019, https://dotesports.com/league-of-legends/news/league-of-legends-generated-1-5-billionrevenue-in-2019, last accessed 2020/04/08.

11. Goslin, A.: Fortnite has 78.3 million monthly players, according to Epic, https://www.polygon.com/fortnite/2018/9/20/17884036/how-many-fortnite-monthlyplayers-2018, last accessed 2020/04/07.

12. Kordyaka, B., Hribersek, S.: Crafting Identity in League of Legends - Purchases as a Tool to Achieve Desired Impressions. In: Proceedings of the 52nd Hawaii International Conference on System Sciences (HICSS-52), Maui, Hawaii, pp. 1506-1515 (2019).

13. Wohn, D.Y., Freeman, G., McLaughlin, C.: Explaining Viewers' Emotional, Instrumental, and Financial Support Provision for Live Streamers. In: Proceedings of the $2018 \mathrm{CHI}$ Conference on Human Factors in Computing Systems, pp. 1-13 (2018).

14. Cai, J., Wohn, D.Y., Mittal, A., Sureshbabu, D.: Utilitarian and Hedonic Motivations for Live Streaming Shopping. In: Proceedings of the 2018 ACM International Conference on Interactive Experiences for TV and Online Video, pp. 81-88 (2018).

15. Riot: League of Legends Is the World Leader in PC Gaming Revenue Since The Start of 2017, https://www.akshonesports.com/article/2017/08/league-legends-world-leader-pcgaming-revenue-since-start-2017, last accessed 2018/02/14.

16. Tassi, P.: Riot Games Reveals "League of Legends" Has 100 Million Monthly Players, https://www.forbes.com/sites/insertcoin/2016/09/13/riot-games-reveals-league-of-legendshas-100-million-monthly-players/, last accessed 2017/09/11.

17. Adinolf, S., Turkay, S.: Toxic Behaviors in Esports Games: Player Perceptions and Coping Strategies. In: Proceedings of the 2018 Annual Symposium on Computer-Human Interaction in Play Companion Extended Abstracts - In: CHI PLAY '18 Extended Abstracts. pp. 365372 (2018).

18. Shores, K.B., He, Y., Swanenburg, K.L., Kraut, R., Riedl, J.: The Identification of Deviance and its Impact on Retention in a Multiplayer Game. ACM Press, pp. 1356-1365 (2014).

19. Kwak, H., Blackburn, J., Han, S.: Exploring Cyberbullying and Other Toxic Behavior in Team Competition Online Games. ACM Press, pp. 3739-3748 (2015). 
20. Barlett, C.P., Gentile, D.A., Chew, C.: Predicting Cyberbullying from Anonymity. Psychology of Popular Media Culture. 5, 171 (2016).

21. Udris, R.: Cyberbullying Among High School Students in Japan: Development and Validation of the Online Disinhibition Scale. Computers in Human Behavior. 41, 253-261 (2014).

22. Lowry, P.B., Moody, G.D., Chatterjee, S.: Using IT Design to Prevent Cyberbullying. Journal of Management Information Systems. 34, 863-901 (2017).

23. Runions, K.C., Bak, M.: Online Moral Disengagement, Cyberbullying, and Cyberaggression. Cyberpsychology, Behavior, and Social Networking. 18, 400-405 (2015).

24. Mueller, M., Heger, O., Kordyaka, B., Kampling, H., Niehaves, B.: Beyond Intuition: Towards a Framework for Empirical-Based Design Theory Building in Design Science Research. In: Proceedings of the 52nd Hawaii International Conference on System Sciences, pp. 5715-5724 (2019).

25. Stahl, B.C., Tremblay, M.C., LeRouge, C.M.: Focus Groups and Critical Social IS Research: How the Choice of Method Can Promote Emancipation of Respondents and Researchers. European Journal of Information Systems 20, 378-394 (2011).

26. Cheung, C.M.K., Wong, R.Y.M., Chan, T.K.H.: Online Disinhibition: Conceptualization, Measurement, and Relation to Aggressive Behaviors. In: Proceedings of International Conference on Information Systems, pp. 1-10. (2016). 